\title{
Proposition d'un test utilisant les mousses aquatiques pour le contrôle radioécologique de l'environnement
}

\author{
Y. BAUDIN-JAULENT et B. DESCAMPS*
}

\begin{abstract}
RESUMÉ
Nous avons vérifié in situ qu' un test utilisant les mousses aquatiques introduction dans l'eau de sacs confectionnés avec du grillage plastique et contenant une petite quantité de mousses - est, en particulier, envisageable pour le contrôle radioécologique d'un complexe minier d'uranium. Ce test possède, en effet, les qualités requises : simplicité de mise en œuvre, fiabilité et faible coût. II peut, en même temps, permettre d'évaluer la pollution minérale due, par exemple, à la présence de métaux lourds, et la pollution organique par des produits comme les dérivés chlorés.
\end{abstract}

\section{ABSTRACT}

Field experiments demonstrated that a test using aquatic mosses (bags made of plastic netting and containing a small amount of moss) could be considered for the radioecological monitoring of a uranium mining complex. This test has the required qualities : easy operation, reliability and low cost. It also makes it possible to assess inorganic pollution due, for instance, to heavy metals, or organic pollution by products such as chlorinated compounds.

\section{INTRODUCTION}

Depuis qu'elles ont permis d'enregistrer la présence de certains radionucléides artificiels issus des retombées des explosions aériennes (avant l'accord de 1963) on sait que les mousses, l'une des deux classes de l'embranchement des bryophytes, sont de bonnes indicatrices des teneurs en radionucléides du substrat - sol et eau - sur et dans lequel elles vivent [1].

Des études expérimentales mettant en jeu des radionucléides comme ${ }^{137} \mathrm{Cs},{ }^{90} \mathrm{Sr},{ }^{54} \mathrm{Mn},{ }^{51} \mathrm{Cr},{ }^{22} \mathrm{Na}$ et ${ }^{60} \mathrm{Co}[6,7,10]$ ont permis d'étudier les modalités de contamination et de décontamination de la mousse aquatique Platyhypnidium riparioïdes (Hedw.) Dix. Des études de terrain ont ensuite permis de confirmer et de vérifier in situ les qualités radioindicatrices et métalo-indicatrices d'au moins quatre espèces de mousses aquatiques : celle déjà citée et Cinclidotus danubicus, Cinclidotus nigricans et Fontinales

* Commissariat à l'énergie atomique, IPSN, DERS, Service d’études et de recherches sur l'environnement, Laboratoire de radioécologie des eaux continentales, 13108 St-Paul lez Durance Cedex. 
antipyretica. Ces études de terrain concernent des radionucléides $-{ }^{137} \mathrm{Cs}$, ${ }^{90} \mathrm{Sr},{ }^{54} \mathrm{Mn}$ - en aval de centrales nucléaires [8-9] et des métaux lourds $\mathrm{Cr}, \mathrm{Cd}, \mathrm{Pb}, \mathrm{Zn}, \mathrm{Cu}, \mathrm{Fe}, \mathrm{Hg}, \mathrm{As}$ - dans des rivières belges, françaises et anglaises $[4,11,12,14,15]$. Compte tenu de leurs caractéristiques écologiques nécessité d'un point de fixation et d'un courant relativement fort - les mousses ne se rencontrent pas partout ; l'idée de les introduire artificiellement est née de cette constatation. Cette implantation a déjà permis de déterminer le niveau de pollution de certaines rivières par le chrome et le cuivre [15] et par des produits chlorés [14].

Dans la présente étude, nous avons effectué un apport exogène de mousses aquatiques dans l'environnement d'un complexe minier - mine et usine d'uranium - de la Cogéma, situé à Lodève. Le but était de vérifier que cette technique pouvait être proposée pour le contrôle des différents radionucléides naturels (principalement le radium) rejetés par ce complexe minier. II est à noter que la méthode de mesure mise en œuvre - la spectrométrie $\gamma \mathrm{Ge}-\mathrm{Li}$ permettait, en même temps, de renseigner sur les autres émetteurs $\gamma$ présents : c'est le cas, en particulier, de ${ }^{137} \mathrm{Cs}$ et du couple ${ }^{144} \mathrm{Ce}+\mathrm{Pr}$, issus des explosions nucléaires aériennes. Ajoutons aussi qu'en utilisant d'autres techniques de mesure (l'activation neutronique, par exemple) on pourrait obtenir, sur ces mêmes mousses, des indications sur leurs teneurs en différents métaux en relation avec les rejets de l'usine.

\section{LE COMPLEXE MINIER DE LODEVE}

Depuis fin 1980, fonctionnent à Lodève dans l'Hérault (France) une mine et une usine de concentration d'uranium d'une capacité annuelle d'environ 1000 tonnes de "yellow cake". Deux rejets liquides sont effectués dans l'environnement :

- l'un est le trop plein de la station de traitement au chlorure de baryum qui a pour fonction d'abaisser la teneur en radium des eaux pénétrant dans cette station à moins de $0,037 \mathrm{~Bq} \cdot \mathrm{I}^{-1}$, concentration maximale admissible de ce radionucléide dans l'eau (fraction soluble);

- l'autre, issu de l'usine, se caractérise par sa charge saline relativement importante.

Le premier rejet se fait dans un ruisseau, le Riviéral (débit moyen de l'ordre de $10 \mathrm{~m}^{3} \cdot \mathrm{h}^{-1}$, venant grossir un autre ruisseau, le Rivernoux, affluent de la rivière Lergue. Le second se fait directement dans la Lergue (débit moyen de $5 \mathrm{~m}^{3} \cdot \mathrm{s}^{-1}$ ), un peu en amont de la confluence du Rivernoux, la Lergue se jetant dans le fleuve Hérault (fig. 1).

Ce complexe minier fait l'objet d'une étude menée par le Département d'études et de recherches en sécurité du Commissariat à l'énergie atomique dans le cadre d'une action soutenue par la Commission des Communautés européennes. Cette étude porte sur la dispersion du radium 226 et son transfert aux espèces vivantes [2]. En parallèle à ces études de transfert, nous nous proposons de vérifier le bien-fondé de l'utilisation des mousses aquatiques comme l'un des moyens possibles de contrôle radioécologique. 


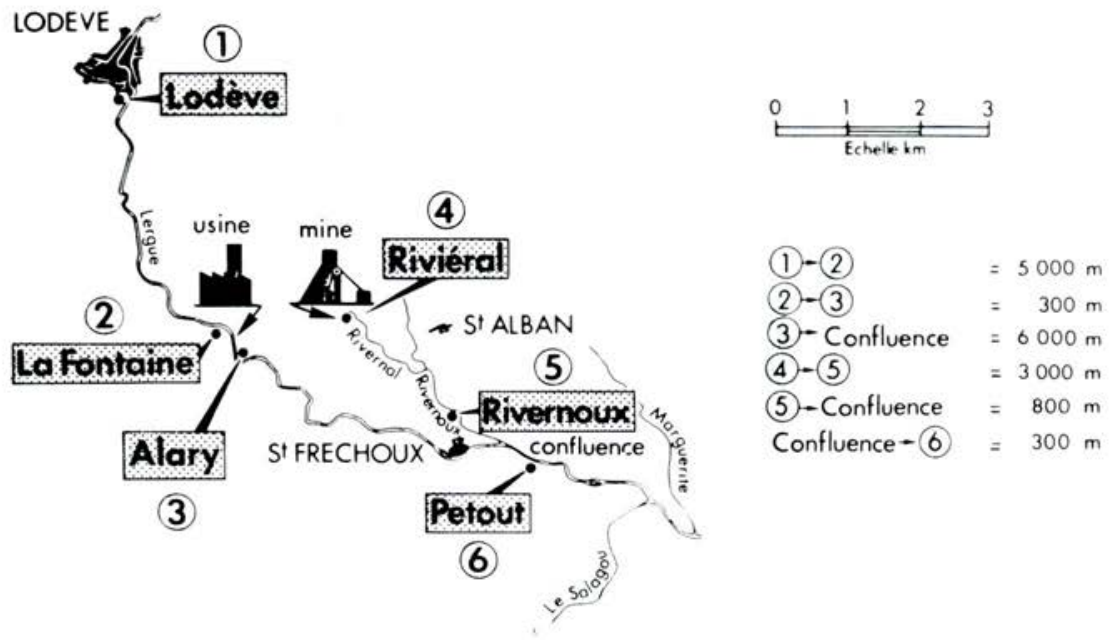

Fig. 1. - Complexe minier de Lodève :

Le réseau hydrographique et les stations utilisées.

\section{MISE EN CEUVRE DU TEST " MOUSSE AQUATIQUE"}

\section{Prélèvement des mousses}

Les deux espèces utilisées : Platyhypnidium riparioïdes (Hedw.) Dix. et Fontinalis antipyretica ont été prélevées dans la Sorgue un peu en aval de sa résurgence à Fontaine-de-Vaucluse, à un endroit où leur prélèvement en quantité relativement importante ne risque pas de provoquer de dommage au site.

\section{Confection des sacs}

Le but à atteindre est de mettre des quantités suffisantes de mousses sous une forme permettant le contact le plus étroit possible entre l'eau et le végétal [4]. Pour cela, des quantités de l'ordre de $200 \mathrm{~g}$ de mousses "fraiches et égouttées" - représentant une grosse poignée lors du prélèvement - ont été placées dans des sacs confectionnés avec du grillage en plastique.

\section{Implantation des sacs sur le site}

L'immersion des sacs a été réalisée dans cinq stations (fig. 1) : La Fontaine (2) en amont immédiat du rejet "usine" ; Alary (3) en aval immédiat de ce même rejet ; Riviéral (4) en aval immédiat du rejet "station de traitement au $\mathrm{Cl}_{2} \mathrm{Ba}^{\prime}$; Rivernoux (5) $3000 \mathrm{~m}$ en aval de la station (4); Petout (6) à l'aval immédiat de la confluence du Rivernoux. 


\section{Temps de contact}

Les cinq temps de contact étudiés n'entrent pas dans un seul et même test ; il y a eu, en fait, trois sous-tests dont les caractéristiques sont données dans le tableau I.

TABLEAU I

Caractéristiques des trois sous-tests réalisés

\begin{tabular}{|l|l|l|l|}
\hline Temps de contact & \multicolumn{1}{|c|}{ Dates } & \multicolumn{1}{|c|}{ Espèces } & \multicolumn{1}{c|}{ Stations } \\
\hline $4 \mathrm{~h}, 24 \mathrm{~h}$ & Octobre 84 & Platyhypnidium & 2.3 .4 .5 .6$. \\
$6 \mathrm{~h}$ & Juin 84 & Platyhypnidium & 4.5. \\
$13 \mathrm{j}, 51 \mathrm{j}$ & Fontinalis & 4.5. \\
& Mars-Avril 84 & Platyhypnidium & 2.4 .5 .6$. \\
\hline
\end{tabular}

\section{Préparation des échantillons et mesures}

Dès leur prélèvement, les mousses sont très soigneusement lavées afin d'éliminer les matières en suspension retenues et les divers organismes présents. Un passage à l'étuve à $110^{\circ} \mathrm{C}$, puis une incinération à $560{ }^{\circ} \mathrm{C}$ pendant $24 \mathrm{~h}$ permettent d'obtenir des cendres. Elles sont intro-

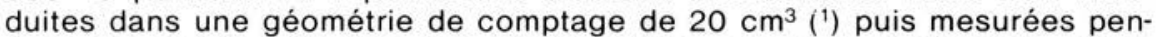
dant une dizaine d'heures par spectrométrie $\gamma \mathrm{Ge}-\mathrm{Li}$ permettant de déterminer tous les émetteurs $\gamma$ présents et en particulier le radium 226 et l'uranium 238. Ces mesures sont faites par le LMEI $\left({ }^{2}\right)$.

\section{RESULTATS}

1. Platyhypnidium riparioïdes (Hedw.) Dix.

Les résultats les plus nombreux ont été obtenus pour cette espèce (tableau II). On peut faire les remarques suivantes :

L'uranium 238 et le radium 226 ont des teneurs qui décroissent selon l'ordre suivant :

$$
\text { Riviéral }>\text { Rivernoux }>>>\text { La Fontaine } \simeq \text { Alary } \simeq \text { Petout } \simeq \text { témoins }
$$

Elles sont donc de bonnes indicatrices des niveaux d'activité (connus d'après notre étude des transferts) de l'eau à ces différentes stations.

Aux stations (4) et (5) on constate que les niveaux d'activité pour $U$ et $R$ a, pris séparément, sont globalement comparables dans les trois sous-tests. Ce résultat est donc la confirmation d'études expérimentales $[5,7,10]$ ayant montré que, dans une eau d'activité donnée, les mousses atteignaient leur niveau d'équilibre en très peu de temps (quelques heures).

(1) Les $200 \mathrm{~g}$ introduits dans les sacs correspondent, en cendres, au remplissage de cette géométrie; le rendement de comptage est ainsi maximal.

(2) Laboratoire de métrologie de l'environnement et d'intervention, Bâtiment 501, Le Bois-des-Rames, 91400 Orsay. 
TABLEAU II

Résultats, en Bq. $\mathbf{k g}^{-1}$ sec, obtenus sur le genre Platyhypnidium

\begin{tabular}{|c|c|c|c|c|c|c|}
\hline \multirow{2}{*}{ Station } & \multicolumn{2}{|c|}{$\begin{array}{r}\text { Temps de contact } 4 \mathrm{~h} \\
24 \mathrm{~h}\end{array}$} & \multicolumn{2}{|c|}{ Temps de contact $6 \mathrm{j}$} & \multicolumn{2}{|c|}{$\begin{array}{r}\text { Temps de contact } 13 \mathrm{j} \\
51 \mathrm{j}\end{array}$} \\
\hline & $U$ & $\mathrm{Ra}$ & $U$ & $\mathrm{Ra}$ & $U$ & $\mathrm{Ra}$ \\
\hline Témoin Sorgue & $41,9 \pm 14,3$ & $31,8 \pm 11,6$ & & & & \\
\hline Témoin Lergue $\left({ }^{1}\right)$ & $49 \pm 11,2$ & $49 \pm 11,2\left({ }^{2}\right)$ & & & & \\
\hline (2) La Fontaine & $37 \pm 12$ & $37 \pm 12$ & & & $34,5 \pm 4,6$ & $16 \pm 16$ \\
\hline & $15 \pm 6,7$ & $15 \pm 6,7$ & & & & \\
\hline (3) Alary & $46 \pm 18$ & $46 \pm 18$ & & & & \\
\hline & $18 \pm 7,4$ & $18 \pm 7,4$ & & & & \\
\hline (4) Riviéral & $341 \pm 47$ & $249 \pm 86$ & $530 \pm 177$ & $819 \pm 273$ & $698 \pm 155$ & $496 \pm 103$ \\
\hline & $647 \pm 62$ & $392 \pm 98$ & & & $1109 \pm 317$ & $634 \pm 317$ \\
\hline (5) Rivernoux & $252 \pm 37$ & $82 \pm 44$ & $428 \pm 78$ & $383 \pm 162$ & $559 \pm 90$ & $188 \pm 71$ \\
\hline & $268 \pm 77$ & $141 \pm 96$ & & & $453 \pm 118$ & $295 \pm 177$ \\
\hline 6) Petout & $50 \pm 25$ & $123 \pm 66$ & & & $195 \pm 56$ & $37 \pm 5,6$ \\
\hline & $44 \pm 19$ & $139 \pm 57$ & & & & \\
\hline
\end{tabular}

(1) L'espèce autochtone, en amont.

(2) Quand les niveaux sont faibles le mesureur ne peut voir de différence entre $U$ et $R a$; ils les donnent à l'équilibre.

- Le rejet de l'usine n'est pas enrichi en U et Ra.

- La dilution de la Lergue est suffisante pour qu'à la station (6), on ne puisse constater, de façon significative, d'augmentation des teneurs en $U$ et $R$ a.

- Entre la station (4) et la station (5) la baisse des teneurs en $U$ et $R a$, tous temps de contact confondus, va de 54 à $67 \%$ pour le radium et de 19 à $59 \%$ pour l'uranium ; ceci est conforme à ce l'on sait pour la fraction dissoute dans l'eau : le radium " disparaît" plus vite que l'uranium.

Quatre autres constatations s'imposent (résultats chiffrés non présentés dans ce document synthétique) :

- Les deux rejets ont des teneurs non enrichies en éléments de la famille du thorium 232 (dosage sur le pic du ${ }^{228} \mathrm{Ac}$ ). La teneur moyenne est de 7,8 Bq. $\mathrm{kg}^{-1} \mathrm{sec}$.

- La famille de l'uranium 235 n'est décelée que dans 5 échantillons sur 49 ; dans ces cinq cas le rapport isotopique $U_{8} / U_{5}$ est normal $(20,7)$.

- Le césium 137 est le seul radionucléide artificiel décelé ; les niveaux sont comparables, compte tenu des variations individuelles, pour tous les échantillons. La moyenne est de 20,6 Bq. kg ${ }^{-1} \mathrm{sec}$.

L'absence de ${ }^{95} \mathrm{Nb},{ }^{95} \mathrm{Zr},{ }^{144} \mathrm{Ce}+\mathrm{Pr}$ indique qu'il n'y a pas eu d'explosion nucléaire aérienne dans les dix-huit mois précédent le test; en effet, ces nucléides sont caractéristiques des retombées jeunes.

\section{Fontinalis antipyretica}

Nous avons fait un test comparatif sommaire avec cette autre espèce, réputée aussi sensible [4]. Le tableau III, comparant les deux espèces 
pour un temps de contact de $6 \mathrm{j}$, montre clairement que Fontinalis antipyretica peut être également utilisée.

TABLEAU III

Comparaisons, pour le temps de contact $6 \mathrm{j}$, entre deux espèces de mousses

\begin{tabular}{|l|l|c|c|}
\hline \multicolumn{1}{|c|}{ Stations } & \multicolumn{1}{|c|}{ Espèces } & Uranium & Radium \\
\hline \multirow{2}{*}{ Témoin Sorgue } & Platyhypnidium & $41,9 \pm 14,3$ & $31,8 \pm 11,6$ \\
& Fontinalis & $45,4 \pm 16,2$ & $45,4 \pm 16,2$ \\
Riviéral & Platyhypnidium & $530 \pm 177$ & $819 \pm 273$ \\
& Fontinalis & $1162 \pm 144$ & $714 \pm 271$ \\
Rivernoux & Platyhypnidium & $428 \pm 78$ & $383 \pm 162$ \\
& Fontinalis & $418 \pm 62$ & $280 \pm 85$ \\
\hline
\end{tabular}

\section{Commentaires}

Dans le tableau IV, nous faisons figurer les différents aspects strictement techniques qu'il est nécessaire de résoudre pour réaliser ce test.

TABLEAU IV

Problèmes techniques inhérents au test

\begin{tabular}{|l|l|}
\hline \multicolumn{1}{|c|}{ Problèmes à résoudre } & \multicolumn{1}{c|}{ Difficultés pour les résoudre } \\
\hline Mise en œuvre du test & \\
- récupération des mousses témoins ; & $\begin{array}{l}\text { aucune } \\
\text { aucune } \\
\text { - confection des sacs ; } \\
\text { - dépôt et enlèvement des sacs ; } \\
\text { entraînement } \\
\text { - lutte contre le colmatage }\end{array}$ \\
$\begin{array}{l}\text { Analyses dép dép matières en suspension } \\
\text { - préparation des échantillons : } \\
\text { spectrométrie } \gamma \text { Ge-Li. }\end{array}$ & $\begin{array}{l}\text { aucune } \\
\text { nécessite une installation spécialisée } \\
\text { surtout si les niveaux sont faibles }\end{array}$ \\
\hline
\end{tabular}

Si le test "mousse aquatique" est envisageable pour le contrôle radioécologique de l'environnement, c'est avant tout parce que les facteurs de concentration (teneur des mousses en Bq. $\mathrm{kg}^{-1} \mathrm{sec} / \mathrm{teneur}$ de l'eau en Bq. (1) $^{-1}$ des mousses sont importants. En fonction des résultats obtenus pendant les quatre années (1980-1984) de l'étude des facteurs de transfert sur le site de Lodève, les plages de valeurs suivantes pour les facteurs de concentration de l'espèce Platyhypnidium peuvent être retenues : radium 1000-6000; uranium 30-300.

Des études expérimentales [10] ont montré que l'adsorption est le facteur essentiel de cette fixation et qu'elle est indépendante de la température. Ceci est très important, car il existe, en général, des différences de température non négligeables entre la Lergue et l'ensemble RiviéralRivernoux (dues au rejet de la station de traitement au chlorure de baryum). 
Après l'intensité de la réponse, sa fiabilité est la caractéristique la plus intéressante.

Elle permet, en effet, d'envisager de construire une abaque qui transforme une teneur dans les mousses en une teneur "représentative" dans l'eau. Tout l'intérêt de ce test réside dans cette possibilité : gommer les fluctuations des teneurs de l'eau (dues aux variations des rejets et des débits naturels), impossibles à enregistrer sans une technologie lourde et onéreuse, par la mesure de l'activité d'un élément intégrateur à réponse intense et fiable. Si la construction d'une telle abaque rencontre deux inconvénients, elle présente surtout plusieurs avantages (tableau V).

TABLEAU V

Inconvénients et avantages pour la construction d'une abaque permettant de passer d'une teneur des mousses à une teneur de l'eau

\begin{tabular}{|c|l|}
\hline \multicolumn{1}{|c|}{ Inconvénients } & \multicolumn{1}{c|}{ Avantages } \\
\hline $\begin{array}{l}\text { - Influence des périodes de relative } \\
\text { décontamination (à étudier). }\end{array}$ & $\begin{array}{l}\text { - Seule l'adsorption est concernée. } \\
\text { - Influence du colmatage (à éviter). }\end{array}$ \\
$\begin{array}{l}\text { - Faibles variations intraspécifiques. } \\
\text { - Indépendance vis-à-vis de la température } \\
\text { de l'eau, donc des saisons. }\end{array}$ \\
\hline
\end{tabular}

La rapidité de la réponse - moins de $24 \mathrm{~h}$ - peut servir, si tel est l'objectif, à étudier un rejet aigu (début, durée, étendue).

\section{CONCLUSION}

Le contrôle de l'environnement aquatique en aval des installations polluantes se heurte, en général, à plusieurs inconvénients : fluctuations trop nombreuses et trop importantes de l'eau, mauvaise représentativité des échantillons de sédiments, difficulté d'échantillonnage pour les poissons et, généralement, charge financière trop lourde, en particulier par le coût des analyses.

C'est ainsi qu'est née la notion d'indicateur biologique. Les poissons puis les algues benthiques [3] ont successivement été proposés comme organismes indicateurs ; récemment, on a démontré que plusieurs espèces de mousses aquatiques étaient plus intéressantes encore pour enregistrer in situ la pollution par les métaux lourds.

Le présent travail démontre que l'on peut utiliser les espèces Platyhypnidium riparioïdes (Hedw.) Dix. et Fontinalis antipyretica, introduites dans des sacs confectionnés avec du grillage plastique, pour le contrôle radioécologique de l'environnement aquatique d'un complexe minier de production d'uranium. Les radionucléides naturels concernés appartiennent à la famille de l'uranium 238 (dont le radium 226). L'intensité, la fiabilité et la rapidité de la réponse au niveau des mousses permettent, si on le désire, de localiser un point de rejet, de suivre son évolution spatiale, de localiser le point de retour à la normale. 
La construction d'une abaque, spécifique du réseau hydrographique étudié, est, cependant, l'aspect le plus intéressant de ce test car elle permet de corréler une teneur des mousses à une teneur "représentative" de l'eau. On peut, ensuite, grâce aux études sur les facteurs de transfert, prévoir les teneurs dans les autres compartiments (sédiments, végétaux, poissons).

L'argument financier, dû à la diminution du nombre des analyses, plaide aussi en faveur de ce test, de même que sa simplicité de mise en œuvre. Citons enfin, dès lors que d'autres méthodes d'analyses sont mises en jeu - activation neutronique ou absorption atomique - la possibilité de contrôle simultané de la pollution en métaux lourds associée à la pollution radioactive.

Pour ce qui concerne le contrôle radioécologique, il est bien clair que ce test est applicable dans tous les lieux où la présence des radionucléides, naturels et artificiels est probable. Cela veut dire qu'il est envisageable dans tous les cours d'eau si l'on s'intéresse aux retombées des explosions nucléaires aériennes et dans certains d'eux si l'on s'intéresse aux rejets radioactifs des installations nucléaires. Dans ce deuxième cas, on peut s'intéresser aussi bien au contrôle d'un complexe minier, et c'est le cas de notre travail, qu'à celui d'une centrale nucléaire ou d'une usine de retraitement. Ce test peut aussi être utilisé dans les recherches sur les transferts des radionucléides, car il donne des renseignements indirects sur l'évolution des radionucléides dissous dans l'eau.

Remerciements : Au Laboratoire de métrologie de l'environnement et d'intervention d'Orsay dirigé par M. Philippot.

\section{BIBLIOGRAPHIE}

[1] BOVARD P. et GRAUBY A. Fixation des radionucléides des retombées atmosphériques par les sphaignes de tourbières, les polytries et les myriophylles. International symposium on radioecological concentration processes, Stockholm, April 25-29 1966. Oxford: Pergamon, 533-537.

[2] DESCAMPS B., FOULQUIER L et BAUDIN-JAULENT Y. L'apport des mesures hydrobiologiques dans l'étude radioécologique d'un site français d'extraction et de traitement d'uranium. In : Management of wastes from uranium mining and milling, Albuquerque, 10-14 May 1982. Vienne: AIEA, 1982, 523-533.

[3] DESCY J.P. Utilisation des algues benthiques comme indicateurs biologiques de la qualité des eaux courantes. In : La pollution des eaux continentales. Incidence sur les biocénoses aquatiques, 2 éd. (PESSON P., Ed.) Paris : GauthierVillars, 1980, 169-194.

[4] EMPAIN A., LAMBINON L., MOUVET $C$ et KIRCHMANN R. Utilisation des bryophytes aquatiques et subaquatiques comme indicateurs biologiques de la qualité des eaux courantes. In : La pollution des eaux continentales. Incidence sur les biocénoses aquatiques, 2. éd. (PESSON P., Ed.) Paris : Gauthier-Villars : 1980, 195-223.

[5] FOULQUIER L. et HEBRARD J.P. Etude expérimentale de la fixation et de la décontamination du sodium 22 par une mousse dulcicole : Platyhypnidium riparioïdes (Hedw.) Dix. Oecol. Plant., 1976, 11 (3) 267-276.

[6] HEBRARD J.P. et FOULQUIER L. Aperçu sur les modalités de la contamination d'une mousse dulcicole, Platyhypnidium riparioïdes (Hedw.) Dix. par le césium 137 et le strontium 90. Rev. Bryol. Lichénol, 1968, 36 (1/2), 219-242. 
[7] HEBRARD J.P. et FOULQUIER L. Introduction à l'étude de la fixation du manganèse 54 par Platyhypnidium riparioïdes (Hedw.) Dix. Rev. Bryol. Lichénol., $1975,41,(1), 35-54$.

[8] KIRCHMANN R. et LAMBINON J. Bioindicateurs végétaux de la contamination d'un cours d'eau par des effluents de centrale nucléaire à eau préssurisée. Evaluation des rejets, de la centrale de la SENA (Chooz, Ardennes françaises) au moyen des végétaux aquatiques et ripicoles de la Meuse. Bull. Soc. roy. Bot. Belg., $1973,106,187-201$.

[9] KIRCHMANN R., LAMBINON J., BONNIJNS-VAN GELDER E. et COLARD J. Utilisation de bioindicateurs à des fins de surveillance des sites nucléaires. In : Environmental surveillance around nuclear installations, Warsaw, 5-9 Nov. 1973 Vienne: AIEA, 1974, 105-118.

[10] MAUREL-KERMARREC A. Cinétique de la fixation et de la désorption d'un mélange de ${ }^{60} \mathrm{Co},{ }^{51} \mathrm{Cr},{ }^{137} \mathrm{Cs},{ }^{54} \mathrm{Mn}$ et ${ }^{22} \mathrm{Na}$ par une mousse (Platyhypnidium riparioïdes (Hedw.) Dix.) et par une hépatique (Chiloscyphus polyanthos (L.) Corda in Opiz) dulçaquicoles. Thèse $3^{\circ}$ cycle. Aix-Marseille, 1984.

[11] MOUVET C. Utilisation des bryophytes aquatiques pour l'étude de la pollution des cours d'eau par les métaux lourds et les radionucléides. Rev. Biol. Ecol. Mediter., 1979, 6, (3/4) 193-204.

[12] MOUVET C. Pollution de l'Amblève par les métaux lourds en particulier le chrome : dosage dans les eaux et les bryophytes aquatiques. Trib. Cebedeau, 1980, 33 , (445), 527-538.

[13] MOUVET C. Accumulation of chromium and copper by the aquatic moss Fontinalis antipyretica L. ex Hedw transplanted in a metal-contaminated river. Environm. Technol. Letters, 1984, 5, 541-548.

[14] MOUVET C., CORDEBAR P. et RIBETTE M. Etude par la méthode des mousses aquatiques de pollutions métalliques multiformes (Saône, Cance, Deume) et de micropollution organique chlorée (Durance). Agence de bassin Rhône-MéditérranéeCorse et Laboratoire d'écologie, Université de Metz, 1984, G. 8415, 76 pages.

[15] SAY P.J., HARDING P.C. and WHITTON B.A. Aquatic mosses as monitors of heavy metal contamination in the river Etherow, Great Britain. Environm. Pollut. (Series B) ; $1981,2,295-307$. 